\title{
Primary care
}

\section{Primary care groups Modernising primary and community health services}

David Wilkin, Therese Dowswell, Brenda Leese

This is the
second in a
series of five
articles

National Primary
Care Research and
Development
Centre, University
of Manchester,
Manchester
M13 9PL
David Wilkin
professor, health
services research
Therese Dowswell
research fellow
Centre for Research
in Primary Care,
University of Leeds,
Leeds LS2 9PL
Brenda Leese
reader
Correspondence to:
D Wilkin
David.Wilkin@
man.ac.uk
Series editor:
David Wilkin

BMJ 2001;322:1522-4
The government's plan for the NHS, published in July 2000 , sets out an ambitious programme of investment, recognising that "the development of primary care services is key to the modernisation of the NHS." Since the founding of the NHS primary care has been one of its greatest strengths but also its weakness. It has provided low cost, easily accessible care, but it has also been characterised by wide variability in quantity and quality, fragmentation, and a lack of coordination. The Labour government's 1997 white paper on the NHS proposed sweeping away the internal market and promoting a culture of collaboration and partnership. ${ }^{2}$

The establishment of primary care groups in England in 1999-which were charged with developing primary and community health services, commissioning hospital services, and improving the health of communities of around 100000 people-represented a radical change in the organisation of primary and community health services. By 2004 all of these groups will become fully fledged primary care trusts, controlling most of the budget for providing health care to the populations that they serve. These organisations, led by local health professionals, will play a vital role in delivering the changes to primary and community services that the government sees as key to modernising the NHS. Within a framework of goals and performance standards set at the national level, the NHS plan asserts that the responsibility for decisions about services should be devolved to those who best understand local needs and circumstances. ${ }^{1}$ In this article, we focus on three key components of the government's strategy for modernising primary and community services: promoting a more efficient use of resources through collaboration and sharing, improving access to primary care, and enhancing the capacity of the workforce.

\section{National tracker survey}

The national tracker survey is a longitudinal survey of 72 of the 481 primary care groups established in England in 1999. ${ }^{3}$ It aims to evaluate their achievements and identify features associated with success in performing their core functions. The first survey was completed in December 1999 and the second in December 2000. Details of the survey were summarised in the first article in this series. ${ }^{4}$ The evidence used in this article is derived from telephone interviews with

\section{Summary points}

Developing primary and community health services is a key component of the government's plans for modernising the NHS

Primary care groups and trusts have a vital part to play in overcoming the variability, fragmentation, and isolation that have been the weaknesses of primary health care in the NHS

Primary care groups and trusts have introduced initiatives to promote greater collaboration between general practices and to share expertise and resources

Improving access to care is an important feature of the modernisation plan, and most groups and trusts are actively promoting access, particularly for people who have been poorly served traditionally

Groups and trusts are tackling staff shortages by using clinical specialists and promoting extended roles for nurses and pharmacists

69 chairs of primary care groups and trusts (97\% response rate), all but four of whom were general practitioners. The interviews were conducted between October and December 2000. ${ }^{5}$

\section{Collaboration and sharing}

The status of general practitioners as independent contractors to the NHS, and the fact that they compete with each other for patients, has encouraged practices to concentrate their efforts on providing services for their own patients. General practice fundholding offered further incentives to improve and extend services within practices but led to accusations of increasing inequalities and inefficient use of resources. However, when incentives have encouraged collaborative working, general practitioners have shown both a willingness and an ability to work together. The development of out of hours cooperatives, ${ }^{6}$ total purchasing pilot sites, ${ }^{7}$ and locality commissioning groups ${ }^{8}$ during the 1990 s illustrated the potential for collaborative 
working in general practice. The establishment of primary care groups and trusts is an attempt to develop a stronger collaborative culture in primary care, to reduce inequalities, and to promote the efficient use of resources through greater sharing of staff and facilities.

Many primary care groups and trusts are promoting greater collaboration by sharing resources between practices (table 1). Some of these schemes to share resources were in place before primary care groups were established and others would have developed without intervention; however, most primary care groups and trusts are actively promoting schemes to facilitate the sharing of resources. Previously, some of these services may only have been available to the patients of fundholding practices. Groups and trusts have initiated schemes to make services available to patients throughout their locality. Although at the time of the survey many developments were still at the planning stage, some groups and trusts identified schemes that they had initiated previously and that had already been implemented (table 1). For example, 12 of the 17 schemes already in place for sharing facilities for minor surgery had been initiated by the group or trust. More than three quarters $(88 \%)$ had initiated at least one scheme for sharing resources between practices.

\section{Improving access to services}

Improving access to primary care services has been a recurring theme in government policy over the past four years, and it is an important component of the NHS plan. ${ }^{1}$ High profile national initiatives such as NHS Direct, walk-in centres, and one stop primary care centres need to be accompanied by local efforts to target poorly served groups, extend surgery opening hours, reduce waiting times for appointments, and develop the roles of nurses and pharmacists to provide care at the first point of contact. In the face of rising demand and increased expectations, primary care groups and trusts are looking for ways to manage demand more effectively and efficiently.

Most primary care groups and trusts have initiated schemes to improve access and manage demand (table 2). Although walk-in centres, often staffed by nurses, have been strongly promoted nationally as a way to provide alternative points of access to health care, they are not widely available or planned. The costs of establishing and operating such centres has tended to restrict them to those groups or trusts that have been awarded additional funding as part of the government's initiative. They may also be difficult to integrate into the existing provision of health care which is practice based. More than half of the groups and trusts surveyed were, however, operating or developing healthy living centres (local centres with a remit to promote health and prevent disease). Many were focusing attention on ways of improving access to existing services by extending surgery opening times and implementing initiatives to reduce waiting times for appointments. Although many of the schemes initiated by groups and trusts were still at the planning stage at the time of the survey, some had already been implemented. Eleven of the 16 schemes already operating to reduce waiting times for appointments had been initiated by groups or trusts.
Table 1 Resources being shared by practices in primary care groups and trusts. ${ }^{5}$ Values are number (percentage)

\begin{tabular}{llcc} 
Resource shared & Groups or trusts sharing resources $(\mathbf{n = 6 9 )}$ & $\begin{array}{c}\text { Groups and trusts that had } \\
\text { initiated existing or planned } \\
\text { schemes ( } \mathbf{n = 6 9 )}\end{array}$ \\
\cline { 2 - 4 } Specialist nurse & Current & Planned & $26(38)$ \\
\hline Counsellor & $30(44)$ & $18(26)$ & $39(57)$ \\
\hline Minor surgery facility & $45(65)$ & $11(16)$ & $30(44)$ \\
\hline Out of hours centre & $17(25)$ & $19(28)$ & $13(19)$ \\
\hline Specialist outreach clinic & $46(67)$ & $12(17)$ & $30(44)$ \\
\hline
\end{tabular}

Table 2 Schemes used to improve access to primary care by groups and trusts. ${ }^{5}$ Values are number (percentage)

\begin{tabular}{|c|c|c|c|}
\hline \multirow[b]{2}{*}{ Scheme } & \multicolumn{2}{|c|}{$\begin{array}{c}\text { Groups or trusts } \\
\text { participating }(n=69)\end{array}$} & \multirow{2}{*}{$\begin{array}{l}\text { Groups and trusts that } \\
\text { had initiated existing or } \\
\text { planned schemes }(n=69)\end{array}$} \\
\hline & Current & Planned & \\
\hline Extended surgery opening hours & $14(20)$ & $17(25)$ & $20(29)$ \\
\hline Reduce waiting times & $16(23)$ & $21(30)$ & $28(41)$ \\
\hline Target poorly served areas or groups & $22(32)$ & $33(48)$ & $43(62)$ \\
\hline Establish walk-in centre & $6(9)$ & $12(17)$ & $8(12)$ \\
\hline Establish healthy living centres & $7(10)$ & $34(49)$ & $25(36)$ \\
\hline
\end{tabular}

Difficulties in accessing primary care services tend to be concentrated among particular groups (for example, homeless people, drug users, refugees, and members of minority ethnic groups) and in particular areas (for example, inner cities, sparsely populated rural areas, and housing estates in deprived areas). The extra resources and innovative services that some fundholding practices were able to implement to improve access may not have targeted the groups and areas with the greatest needs. Primary care groups and trusts may be better able to identify needs, target resources, and develop services for their local areas. Fifty three $(77 \%)$ of those surveyed had schemes operating or planned to improve access for poorly served groups or areas.

\section{Enhancing workforce capacity}

The success of national and local strategies in improving the provision of primary and community health services will depend to a substantial extent on the capacity and flexibility of the workforce. The NHS plan emphasises the need to break down "old fashioned demarcations between staff" to improve services. ${ }^{1}$ The need for staff to develop new skills and take on new roles is made more pressing by widespread problems in recruiting and retaining sufficient numbers of

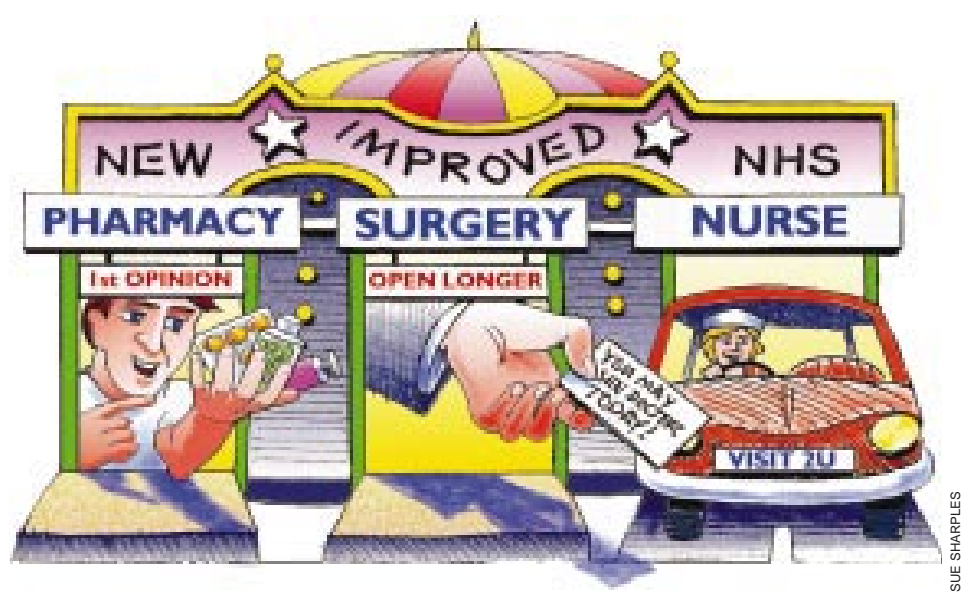


Table 3 Schemes implemented to enhance workforce in primary care groups and trusts. ${ }^{5}$ Values are number (percentage)

\begin{tabular}{|c|c|c|c|}
\hline \multirow[b]{2}{*}{ Scheme } & \multicolumn{2}{|c|}{ Groups and trusts participating $(\mathrm{n}=69)$} & \multirow{2}{*}{$\begin{array}{l}\text { Groups and trusts that had } \\
\text { initiated existing or } \\
\text { planned schemes }(n=69)\end{array}$} \\
\hline & Current & Planned & \\
\hline $\begin{array}{l}\text { Employ salaried general } \\
\text { practitioners }\end{array}$ & $19(28)$ & $22(32)$ & $25(36)$ \\
\hline $\begin{array}{l}\text { Recruit and retain other health } \\
\text { professionals }\end{array}$ & $13(19)$ & $21(30)$ & $25(36)$ \\
\hline Develop GP clinical specialists & $14(20)$ & $42(61)$ & $44(64)$ \\
\hline Employ nurse practitioners & $19(28)$ & $33(48)$ & $38(55)$ \\
\hline Extend role of pharmacists & $18(26)$ & $23(33)$ & $29(42)$ \\
\hline
\end{tabular}

general practitioners and other health professionals. Six months after being established, 19\% of groups and trusts reported problems with recruiting and retaining general practitioners, and 40\% said that they had an insufficient number of practice nurses. ${ }^{3}$

By their second year, most groups and trusts had begun to implement schemes to tackle recruitment and retention or to enhance the capacity of the workforce (by having health professionals acquire new skills or extend their roles), or both (table 3). Recruiting general practitioners to salaried positions under the personal medical services pilot scheme ${ }^{9}$ was often associated with initiatives aimed at groups or areas that were poorly served. Similarly, schemes to develop the roles of nurse practitioners and pharmacists were often prompted by the need to find better ways of managing increasing demand and rising expectations.

\section{Are services being modernised?}

Whether many of the targets set out in the government's national plan for the NHS are achieved depends on whether primary care groups and trusts can modernise primary care and community services. Understandably, their first year was largely taken up with establishing an infrastructure and finding ways to work together as effective organisations. ${ }^{3810}$ By the end of their second year, they had begun to play an active role in implementing national policy and shaping local services. It is still too early to expect to see large improvements in services for most patients despite the recent announcement of increased funding for the NHS as a whole and primary care in particular. ${ }^{11}$

Nevertheless, ministers and the NHS Executive can be pleased with the performance of groups and trusts so far. Although progress is by no means uniform, most groups and trusts are sharing resources and expertise between practices, introducing initiatives to improve access to primary care and manage demand, and looking for ways of sustaining and developing the capacity of their workforce. Many of these initiatives are still at the planning stage, but some groups have already made changes that have the potential to deliver a better service.

One of the perceived deficiencies of the internal market of the 1990s was its failure to address inequalities. Primary care groups and trusts are able to take a more strategic approach to developing services because they have the responsibility to deliver better and more equitable services and they control their own budget. Not all of them are doing so, but the fact that many were targeting initiatives at poorly served groups or areas is encouraging. The many schemes for sharing resources between practices-as a means of ensuring greater equity and access to a wider range of servicesare also important. General practice fundholding often had the opposite effect: it restricted access to services to the patients of particular practices regardless of need.

Primary care groups and trusts are making good progress in improving primary care, but there are a number of reasons to be cautious. Firstly, the evidence is drawn almost entirely from reports provided by those who are closely involved with these organisations. In this article we have drawn entirely on the accounts provided by chairs of groups and trusts; these are general practitioners who will inevitably be anxious to promote their own achievements. Thus, there is a need for direct evidence of the impact on services and the experiences of patients.

Secondly, while we have highlighted their successes in implementing national policy, some of those interviewed expressed concern that insufficient attention was being paid to local priorities. Individual primary care groups and trusts inherited widely varying populations and practices, and it is appropriate that their policies and priorities should reflect these differences. ${ }^{12}$ This raises questions about the extent to which both national policies and those of the group or trust reflect the priorities of ordinary general practitioners, nurses, and other health professionals. ${ }^{13}$

Thirdly, although most primary care groups and trusts were implementing some initiatives, some seemed to have made relatively little progress. And lastly, as emphasised previously, ${ }^{4}$ the combination of tightly constrained managerial capacity and a heavy workload, arising from the organisational changes associated with mergers and the transition to trust status, raises concerns about the capacity of groups and trusts to devote sufficient resources to changes that will directly affect services to patients.

Funding: The national tracker survey is funded by the Department of Health and carried out by the National Primary Care Research and Development Centre in collaboration with the King's Fund.

Competing interests: None declared.

1 Secretary of State for Health. The NHS plan: a plan for investment, a plan for reform London: Stationery Office, 2000. (Cm 4818-I)

2 Secretary of State for Health. The new NHS: modern, dependable. London: Stationery Office, 1997. (Cm 3807.)

3 Wilkin D, Gillam S, Leese B, eds. The national tracker survey of primary care groups and trusts: progress and challenges 1999/2000. Manchester: National Primary Care Research and Development Centre, King's Fund, 2000. (Available at www.npcrdc.man.ac.uk/pages/research/pcg.htm.)

4 Wilkin D, Gillam S, Smith K. Tackling organisational change in the new NHS. BMJ 2001;322:1464-7.

5 Wilkin D, Gillam S, eds. The national tracker survey of primary care groups and trusts 2000/2001: modernising the NHS? Manchester: National Primary Care Research and Development Centre (in press).

6 Hallam L, Reynolds M. GP out-of hours co-operatives. In: Salisbury C, Dale J, Hallam L, eds. 24 hour primary care. Abingdon: Radcliffe Medical Press, 1999:63-91.

7 Mays N, Goodwin N, Killoran A, Malbon G. Total purchasing. A step towards primary care groups. London: King's Fund, 1998.

8 Regen E, Smith J, Shapiro J. First off the starting block. Lessons for GP commissioning pilots for primary care groups. Birmingham: Health Services Management Centre, University of Birmingham, 1999.

9 Department of Health. Personal medical services pilots under the new NHS (Primary Care) Act 1997-a comprehensive guide. London: DoH, 1997.

10 Audit Commission. The PCG agenda. Early progress of primary care groups in "the new NHS." London: Audit Commission, 2000.

11 Hunter M. Doctors give guarded response to $£ 100 \mathrm{~m}$ for GP services. BMJ 2001;322:696.

12 Majeed A, Bardsley M, Morgan D, O'Sullivan C, Bindman A. Cross sectional study of primary care groups in London: association of measures of socioeconomic and health status with hospital admission rates. $B M J$ 2000;321:1057-60.

13 Lucas K, Bickler G. Altogether now? Professional differences in the priorities of primary care groups. J Public Health Med 2000;22:211-5. 